\title{
ALMA Explorations of Warm Dense Molecular Gas in Nearby LIRGs
}

\author{
C. Kevin Xu \\ Infrared Processing and Analysis Center, \\ MS 100-22, California Institute of Technology, \\ Pasadena, CA 91125 \\ email: cxu@ipac.caltech.edu
}

\begin{abstract}
We present results of ALMA (Cycle-0) observations of the CO (6-5) line emission and the $435 \mu \mathrm{m}$ continuum of two nearby luminous infrared galaxies (LIRGs) NGC 34 (a major merger with an AGN) and NGC 1614 (a minor merger with a circum-nuclear starburst). Using receivers in the highest frequency ALMA band available (Band-9), these observations achieved the best angular resolutions $\left(\sim 0^{\prime \prime} .25\right)$ for ALMA Cycle- 0 observations and resolved for the first time distributions of warm dense molecular gas $\left(\mathrm{n}>10^{5} \mathrm{~cm}^{-3}\right.$, T >100 K) in LIRGs with spatial resolutions better than $100 \mathrm{pc}$. Our ALMA data show a very tight correlation between the $\mathrm{CO}(6-5)$ line emission and the $435 \mu \mathrm{m}$ dust continuum emission, suggesting the warm dense molecular gas dominates the ISM in the central kpc of LIRGs, and gas heating and dust heating in the warm dense gas cores are strongly coupled. On the other hand, we saw very different spatial distributions and kinematic properties of warm dense gas in the two LIRGs, indicating that physical conditions in the ISM can be very different in different LIRGs.
\end{abstract}

Keywords. galaxies: interactions - galaxies: evolution - galaxies: starburst - galaxies: general galaxies: active - submillimeter: galaxies

\section{Introduction}

Luminous infrared galaxies (LIRGs) with $\mathrm{L}_{\mathrm{IR}}[8-1000 \mu \mathrm{m}]>10^{11,5} \mathrm{~L}_{\odot}$, including ultraluminous infrared galaxies (ULIRGs: $\mathrm{L}_{\mathrm{IR}}>10^{12} \mathrm{~L}_{\odot}$ ) are mostly advanced mergers (?). Extensive surveys of CO rotation lines in low J transitions such as CO (1-0) at $2.6 \mathrm{~mm}$ and $\mathrm{CO}(2-1)$ at $1.3 \mathrm{~mm}$ have found very large amounts of molecular gas (up to a few times $10^{10} \mathrm{M}_{\odot}$ ) in the central kpc of (U)LIRGs (Scoville et al. 1989; Sanders et al. 1991). This gas, funneled into the nuclear region by the gravitational torque in a merger (Barnes \& Hernquist 1996; Hopkins et al. 2009), provides fuel for the nuclear starburst and/or AGN. However, due to the heavy dust extinction for the UV/optical/NIR observations and the lack of high angular resolution FIR/sub- $\mathrm{mm} / \mathrm{mm}$ observations, it is still not very clear how the different constituents (i.e., gas, dust, stars, and black holes) in (U)LIRG nuclei interplay with each other. Some studies (Scoville et al. 1997; Downes \& Solomon 1998; Bryant \& Scoville 1999; Gao et al. 2001) suggest that much of the low J CO luminosities may be due to the emission of diffuse gas not closely related to the active star formation regions. Indeed, single dish and interferometry $\mathrm{mm}$ and submm observations have found that the intensities and spatial distributions of star formation in (U)LIRGs correlate significantly stronger with those of higher $\mathrm{J}$ CO lines (with upper level $\mathrm{J} \geqslant 3$ ), which probe warmer and denser gas than low J lines (Yao et al. 2003; Iono et al. 2004; Wang et al. 2004; Wilson et al. 2008; Iono et al. 2009; Sakamoto et al. 2008, 2013; Xu et al. 2014; Lu et al. 2014). 

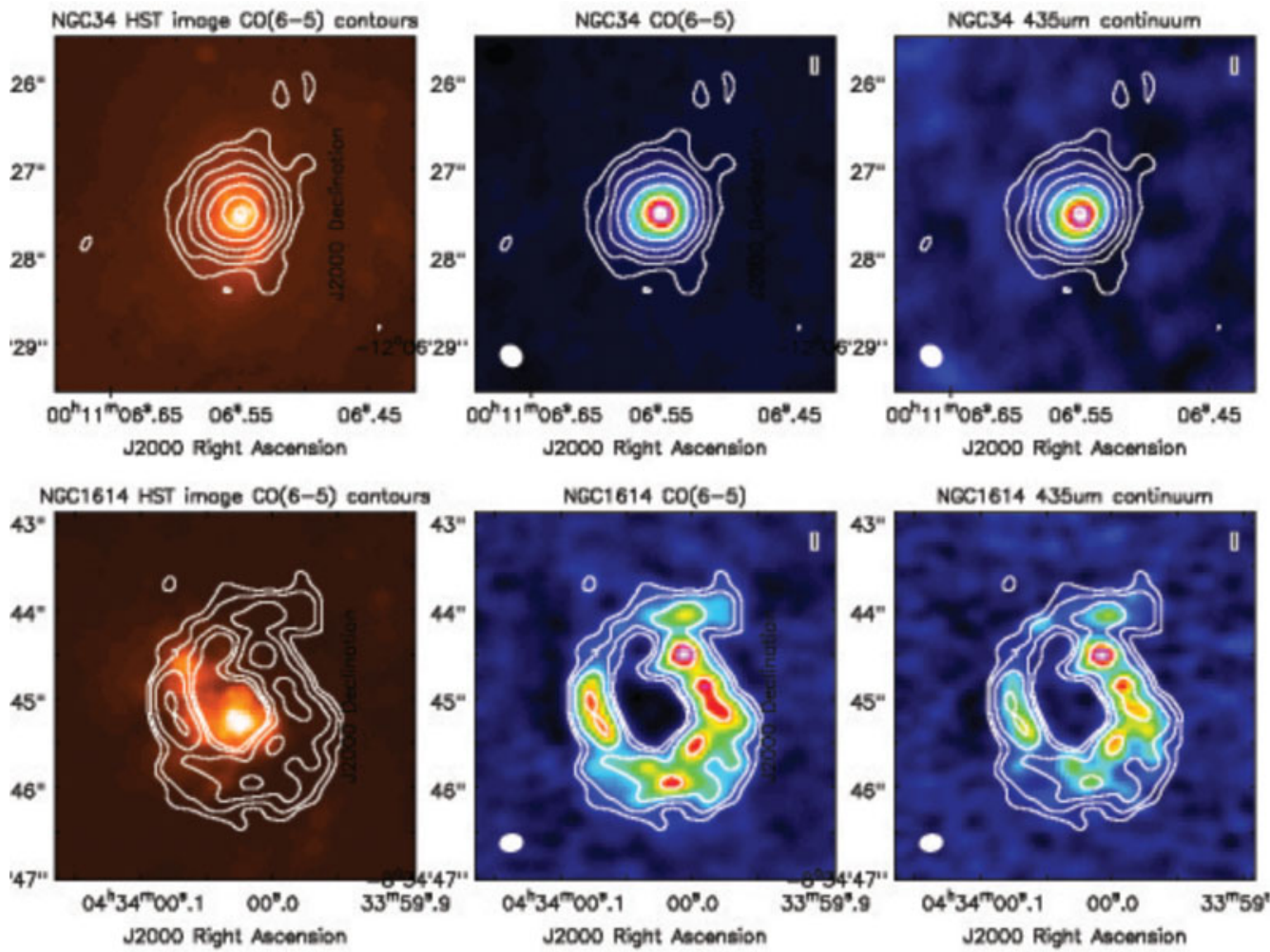

NCC1614 435um continuum

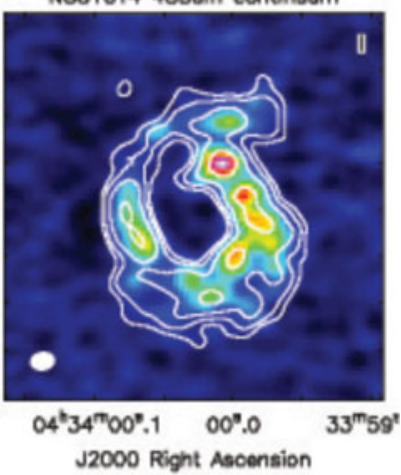

Figure 1. Upper-left: The HST V-band image of NGC 34 overlaid by contours of the integrated CO (6-5) line emission. The contour levels are $[1,2,4,8,16,32,50] \times 3.6 \mathrm{Jy} \mathrm{km} \mathrm{s}^{-1}$ beam $^{-1}$. Upper-mid: Image and contours of the integrated CO (6-5) line emission of NGC 34. Upper-right: Image of the continuum of NGC 34 overlaid by contours of the integrated $\mathrm{CO}(6-5)$ line emission. Lower-left: The HST V-band image of NGC 1614 overlaid by contours of the integrated CO (6-5) line emission. The contour levels are $[1,2,4,8] \times 2.7 \mathrm{Jy} \mathrm{km} \mathrm{s}^{-1}$ beam $^{-1}$. Lower-mid: Image and contours of the integrated CO (6-5) line emission of NGC 1614. Lower-right: Image of the continuum of NGC 1614 overlaid by contours of the integrated CO (6-5) line emission.

\section{NGC 1614 and NGC 34: A Tale of Two LIRGs}

In order to study the warm dense gas in nuclear regions of (U)LIRGs, we observed the CO (6-5) line emission (rest-frame frequency $=691.473 \mathrm{GHz}$ ) and associated dust continuum emission in two nearby (U)LIRGs (NGC 34 and NGC 1614) using the Band 9 receivers of the Atacama Large Millimeter Array (ALMA; Wootten \& Thompson 2009). Both NGC 34 and NGC 1614 were chosen for these early ALMA observations, among the complete sample of 202 LIRGs of the Great Observatories All-sky LIRG Survey (GOALS; Armus et al. 2009), because of their close proximity ( $\mathrm{D}<85 \mathrm{pc}$ ) and bright CO (6-5) line flux $\left(\mathrm{f}_{\mathrm{CO}(6-5)} \gtrsim 1000 \mathrm{Jy} \mathrm{km} \mathrm{s}^{-1}\right)$ observed in the Herschel SPIRE FTS survey of GOALS galaxies (angular resolution: 30"; van der Werf et al. 2010; Lu et al. 2014). This enables high signal-to-noise-ratio ALMA observations of warm gas structures with linear resolutions of $\lesssim 100 \mathrm{pc}$ for the given angular resolutions of $\sim 0^{\prime \prime} .25$. Further, both LIRGs have declination angles close to the latitude of the ALMA site, therefore the Band 9 observations are affected by minimal atmospheric absorption when being carried out near transit. 
Table 1. Results of ALMA Observations

\begin{tabular}{lccccc}
\hline name & beam & $\mathrm{f}_{\mathrm{CO}(6-5)}$ & $\mathrm{f}_{\mathrm{CO}(6-5)}^{\mathrm{ALMA}} / \mathrm{f}_{\mathrm{CO}(6-5)}^{\mathrm{Hsch}}$ & $\mathrm{f}_{435 \mu \mathrm{m}}$ & $\mathrm{f}_{435 \mu \mathrm{m}}^{\mathrm{ALMA}} / \mathrm{f}_{435 \mu \mathrm{m}}^{\mathrm{Hsch}}$ \\
\hline \multicolumn{5}{c}{$\left(\mathrm{Jy} \mathrm{km} \mathrm{s}^{-1}\right)$} \\
\hline NGC 34 & $0^{\prime \prime} .26 \times 0^{\prime \prime} .23$ & $1004 \pm 151$ & $1.07 \pm 0.18$ & $275 \pm 41$ & $0.53 \pm 0.08$ \\
NGC 1614 & $0^{\prime \prime} .26 \times 0^{\prime \prime} .20$ & $898 \pm 153$ & $0.63 \pm 0.12$ & $269 \pm 46$ & $0.32 \pm 0.06$ \\
\hline
\end{tabular}

In Fig 1 we compare the ALMA images with the HST V-band images (Malken et al. 1998) of the two LIRGs. We obtained the following results from the ALMA observations:

- The CO (6-5) line emission and the $435 \mu \mathrm{m}$ continuum in the central kpc of both LIRGs are well detected and resolved by the ALMA observations.

- In both LIRGs, the CO (6-5) line emission and the continuum correlate tightly with each other. This suggests that the warm dense molecular gas (probed by the CO (6-5) line emission) dominates the ISM in the central kpc of LIRGs, and gas heating and dust heating in the warm dense gas cores are strongly coupled.

- The CO (6-5) line emission shows very different morphologies in the central kpc of the two LIRGs. In NGC 34, a major-merger with a weak AGN, it is concentrated in a nuclear disk of $\mathrm{r} \sim 100 \mathrm{pc}$, which is $\sim 6$ times smaller than the CO (1-0) disk (Fernandez et al. 2014). In NGC 1614, a minor-merger without any detectable AGN, the CO (6-5) line emission is in a circum-nuclear ring $(100 \lesssim \mathrm{r} \lesssim 350 \mathrm{pc})$ with a central hole.

- In the CO (6-5) line profile of NGC 34, a significant emission feature is detected on the redshifted wing, coincident with the frequency of the $\mathrm{H}^{13} \mathrm{CN}$ (8-7) line emission which is a very high critical density isotopic molecular line. However, it cannot be ruled out that the feature is due to an outflow of warm dense gas with a mean velocity of $400 \mathrm{~km} \mathrm{~s}^{-1}$.

- The non-detections of the nucleus in both the CO (6-5) and the $435 \mu \mathrm{m}$ continuum rule out, with relatively high confidence, a Compton-thick AGN in NGC 1614.

- Comparisons between the ALMA maps and high resolution radio continuum and Pa $\alpha$ maps of NGC 1614 show that the local correlation (on the linear scale of $\sim 100 \mathrm{pc}$ ) between $\Sigma_{\text {Gas }}$ and $\Sigma_{\mathrm{SFR}}$ (i.e. the Kennicutt-Schmidt law) is severely disturbed. In particular, the nucleus has a lower-limit of the $\Sigma_{\mathrm{SFR}}-$ to- $\Sigma_{\text {Gas }}$ ratio about an order of magnitude above the nominal value in the standard Kennicutt-Schmidt law.

\section{Acknowledgements}

This paper makes use of the following ALMA data: ADS/JAO.ALMA\#2011.0.00182.S. ALMA is a partnership of ESO (representing its member states), NSF (USA) and NINS (Japan), together with NRC (Canada) and NSC and ASIAA (Taiwan), in cooperation with the Republic of Chile. The Joint ALMA Observatory is operated by ESO, AUI/NRAO and NAOJ.

\section{References}

Armus, L., et al. 2009, PASP, 121, 599

Barnes, J. \& Hernquist, L. 1996, ApJ, 471, 115

Bryant, P. M. \& Scoville, N. Z. 1999, AJ, 117, 2632

Downes, D. \& Solomon, P. M. 1998, ApJ, 507, 615

Fernandez, X., Petric, A. O., Schweizer, F., \& van Gorkom, J. H. 2014, arXiv: 1401.1821 
Gao, Y., Lo, K. Y., Lee, S.-W., \& Lee, T.-H. 2001, ApJ, 548, 172

Hopkins, P. F., et al. 2009, ApJ, 691, 1186

Iono, D., Ho, P. T. P., Yun, M. S., et al. 2004, ApJ, 616, L63

Iono, D., Wilson, C. D., Yun, M. S., et al. 2009, ApJ, 695, 1537

Lu, N., Zhao, Y., Xu, K. C., et al. 2014, ApJ, 787, L23

Malken, M. A., et al. 1998, ApJS, 117, 25

Sakamoto, K., Aalto, S., Costagliola, F., et al. 2013, ApJ, 764, 42

Sakamoto, K., Wang, J., Wiedner, M. C., et al. 2008, ApJ, 684, 957

Sanders, D. B., Scoville, N. Z., \& Soifer, B. T. 1991, ApJ, 370, 158

Scoville, N. Z., Sanders, D. B., Sargent, A. I., et al. 1989, ApJ, 345, L25

Scoville, N. Z., Yun, M. S., \& Bryant, P. M. 1997, ApJ, 484, 702

van der Werf, P. P., Isaak, K. G., Meijerink, R., et al. 2010, A\&A, 518, L42

Wang, J., Zhang, Q., Wang, Z., et al. 2004, ApJ, 616, L67

Wilson, C. D., Petitpas, G. R., Iono, D., et al. 2008, ApJS, 178, 189

Wootten, A. \& Thompson, A. R. 2009, IEEE, 97, 1463

Xu, C. K., Cao, C., Lu, N., et al. 2014, ApJ, 787, 48

Yao, L., Seaquist, E. R., Kuno, N., \& Dunne, L. 2003, ApJ, 588, 771 